\title{
(Re)producción de estereotipos de género en el BudismoSoka argentino
}

(Re)production of gender stereotypes in Argentinean SokaBuddhism

Guido Alejo,Sciurano* (CIS-IDES/CONICET, CEDES, IIGG-UBA)

Manuel,Melamud**(IIGG-UBA)

Micaela Belen, Alquezar ***(IIGG-UBA, HUB)

\section{Resumen}

El presente trabajo describe y analiza el budismoSoka en Argentina para reponer de qué modo y mediante qué mecanismos institucionales se (re)producen allí estereotipos de género. La investigación comprendió tres años de observación participante (2018-2020) en múltiples focos etnográficos en el área metropolitana de Buenos Aires, análisis hermenéutico documental y una serie de entrevistas en profundidad a miembros $(n=25)$. El artículo contribuye a informar con datos institucionales/organizacionales los debates sobre nuevas espiritualidades y poner en primer plano los mecanismos de (re)producción material y simbólica de diferencias y desigualdades entre varones y mujeres practicantes de este budismo.

Palabras claves: budismo; análisis institucional; género; estereotipos; Argentina.

\begin{abstract}
This paper describes and analyses the Soka Buddhism in Argentina with the aim of studying gender stereotypes and the specific institutional mechanisms through which they are (re)produced. The research involved three years of participant observation (20182020) in multiple ethnographic spaces in the Buenos Aires metropolitan area, documentary hermeneutic analysis and a series of in-depth interviews with members

*Becario del CONICET.Desarrolla tareas de investigación en el IDES -Programa de Ciudadanía y Derechos Humanos-, el CEDES y el IIGG-UBA. Se especializa en temas de género, sexualidad, cultura y salud.

**Licenciado en Sociología UBA, investigador del IIGG/UBA, integrante del GEAP-IIGG, docente UBA. Se especializa en estudios sociológicos de espiritualidades nueva era.

${ }^{* * *}$ Licenciada en Sociología por la Universidad de Buenos Aires y maestranda en GenderStudies HUB (Humboldt UniversitätzuBerlin). Integrante del Grupo de Estudios Sobre Acciones en Público (GEAP-IIGG). Trabaja en teoría sociológica, género y nuevas espiritualidades.
\end{abstract}


$(n=25)$. The main contributions are to enrich the debates on new spiritualities with institutional/organizational data, and to bring to the fore the mechanisms of material and symbolic (re)production of differences and inequalities among men and women who practice this Buddhism.

Keywords: Buddhism; institutional analysis; gender; stereotypes; Argentina.

Fecha de recepción:Junio 2021

Fecha de aprobación: Diciembre 2021

\section{Introducción: Budismo del Sutra del loto y género en el budismo ${ }^{1}$}

Tributario de las enseñanzas impartidas por Siddharta Gautama en la India del s. V a.C., el budismo del Sutra del loto o budismo Nichiren -que también llamaremos budismo Soka en virtud de la forma nativa de nombrar- constituye una reapropiación y actualización japonesa de una filosofía de vida que atravesó antes el sudeste asiático y China. El itinerario se valió principalmente de la transmisión oral y, como puede verse a partir de los distintos budismos existentes, se fue transformando en cada una de sus paradas y a lo largo del tiempo (Puig Zhamaganov, 2011). NichirenDaishonin fue quien, en Japón y durante parte del siglo XIII, se dio a la tarea de estudiar los Sutras (enseñanzas sucintas a través de las cuales tenía lugar la propagación de esta filosofía) arribando a la conclusión que da origen a la práctica que nos ocupa, a saber, que el Sutra del loto representa, condensa e implica la totalidad de las enseñanzas de su fundador hindú. Este se caracteriza por considerar que toda persona posee la capacidad de alcanzar la iluminación; el estado de budeidad sería, pues, una posibilidad en la vida de cualquier individuo.

Distanciándose de otras prácticas budistas que cuentan con figuras jerárquicas con acceso exclusivo a estados místicos y sagrados de iluminación, el budismo del Sutra del Loto propone una idea democrática que resulta novedosa dentro de las tradiciones orientales. Sin sumos prelados, monjes privilegiados ni casta sacerdotal, cualquier persona que se involucre con las actividades de la práctica puede acceder al estado de iluminación ${ }^{2}$. Esto es así, ya que desde los preceptos básicos del budismo Soka el estado de budeidad es una posibilidad latente y potencial que se encuentra en cada uno de los miembros y practicantes. Cualquier persona que entone la ley Mística -

\footnotetext{
${ }^{1}$ Este estudio se desarrolló con el financiamiento del proyecto "Modos alternativos de vida" (PICT 2016-4634) del MINCyT-Argentina, con sede en el Instituto de Investigaciones en Ciencias Sociales "Gino Germani" (IIGG) de la Facultad de Ciencias Sociales de la Universidad de Buenos Aires (UBA).

2 La postura doctrinaria alusiva a esta máxima y a la dignidad humana puede encontrarse en varias fuentes nativas, entre las cuales figuran: Daishonin (2009), Ikeda (1982), Makiguchi (2011).
} 
NamMyohoRengeKyo- podrá iluminarse. Esta igualdad se articula como consecuencia de otro de los pilares fundamentales del Sutra del Loto: la dignidad humana. Sin hacer una lectura de las acciones humanas en términos de éxitos o fracasos trascendentales que se alejen de la vida cotidiana, la filosofía en cuestión entiende que cualquier sujeto parte de las mismas posibilidades debido al hecho de estar vivo. La posibilidad de expandir la paz mundial y de concebir a la propia vida como una lucha contra obstáculos que termina fortaleciendo al decurso vital de cada individuo dan cuenta de esa motivación endógena que hace hincapié en la vida cotidiana, aquella que, por el mero hecho de existir, ha de ser tratada de forma digna y sin distinciones ni desigualdades (Ikeda, 1984).

La propuesta de Daishonin atravesó un derrotero histórico irregular, con altos y bajos de visibilidad e institucionalización, siempre al calor de la coyuntura política. A nuestros fines basta con señalar que el budismo Nichiren encontró nuevo impulso gracias a un director de escuela japonés que lo unificó a su propuesta pedagógica hacia 1930. Fueron Makiguchi y su discípulo, Toda, quienes fundaron la organización laica SokaKyoiku Gakkai ${ }^{3}$ (Sociedad Educativa para la Creación del Valor), refundada con el nombre de Soka Gakkai luego de la Segunda Guerra Mundial. La proyección internacional de la institución se dio tras un cambio en su dirección en la década de 1960, a partir del cual pasó a ser presidida por su líder actual Daisaku lkeda. Con la experiencia de la devastación bélica como pasado reciente y la Guerra Fría como trasfondo, Ikeda promovió un perfil pacifista y antinuclear que trascendió las fronteras de la isla. Hoy en día la Soka Gakkai Internacional (a partir de aquí SGI) tiene presencia en 192 países, entre los cuales figura Argentina (SGIAR).

Habida cuenta de esta breve presentación de la organización, y para adentrarnos en el estudio aquí propuesto, es preciso recomponer el contexto de discusión académica en el cual se inserta. Es insoslayable la importancia que la comunidad japonesa -así como la SGI- comenzó a cobrar en el siglo XX dentro de la Argentina, en particular en lo que hace a la consolidación de ciertas prácticas, filosofías y actividades vinculadas a un modo de vida religioso proveniente de Oriente. Recuperamos en este punto a Gancedo (2015), quien lo conceptualiza como una diáspora. A partir de este concepto es posible comprender los grandes desplazamientos de personas desde un lugar de origen a otro de recepción, sin dejar de tener en cuenta el surgimiento de una conciencia y comportamiento que es propio de un grupo deslocalizado e intervenido por distintos espacios simultáneamente. A partir de allí el autor comprende que el emplazamiento de la SGI en el territorio argentino puede ser leído como una estrategia de revalorización étnica y/o religiosa, así como también en tanto medio para obtener visibilidad en el nuevo territorio habitado.

Ahora bien, el advenimiento del budismo Soka en la Argentina a partir de los 60' está íntimamente ligado a la llegada de una oleada de nuevas espiritualidades, en

${ }^{3}$ Fundada como una Sociedad Educacional para la Creación de Valores, la institución pasó a tener el nombre que hoy se le conoce (Soka Gakkai) en 1945, bajo el mandato de Josei Toda, su segundo presidente. 
general, provenientes de países asiáticos. Trabajos como el de Semán y Viotti (2015) así como el de Carozzi (1999) dan cuenta de un proceso a partir del cual se masificaron y comenzaron a propagarse nuevas tendencias filosóficas y religiosas que pasaron a formar parte del vasto campo de la espiritualidad. Con ejes puestos en el individuo, la autonomía y la negación de toda autoridad externa al sujeto, estas prácticas de la nueva era tienden a convenir un espacio de creciente movilización e importancia en la cultura contemporánea. Excediendo al propio campo de la religión, que tanto la sociología como la antropología en Latinoamérica han construido como objetos diferenciados (Lehmann y Montalvo, 2004), estas espiritualidades dotan de nuevos sentidos a la vida social en aquellos lugares en los que echan raíces y han favorecido abordajes interdisciplinarios. Complementando estos estudios, consideramos que más allá del individuo y la dimensión filosófica de la práctica, el análisis desde una perspectiva institucional puede aportar datos sustantivos para comprender estos fenómenos emergentes.

En el caso particular del budismo del Sutra del loto en América Latina, algunos autores como Welsch (2018) y May May (2016) han indagado sobre la capacidad de adaptación que tienen sus prácticas e instituciones para insertarse eficazmente en contextos tan disímiles a los de su origen. La primera enumera algunas de las características que habilitan al budismo Soka a trasplantar sin dificultades sus principios básicos al suelo argentino, a saber: la simpleza y eficiencia de la práctica, la posibilidad de socialización que los diferentes espacios internos del budismo permiten, la difusión permanente de actividades, y la preexistencia de algunas espiritualidades orientales en el país. Por otro lado, May May enfatiza algunos sedimentos particulares de la SGI para entender la potencia que tiene a la hora de integrarse en nuevas regiones. Atiende, en primer término, a la existencia de un paralelismo entre los valores de ciertas transformaciones socioculturales acaecidas en algunas áreas mexicanas con aquellos que pregona la institución. Asimismo, destaca la capacidad que tiene la institución para interpelar dos elementos claves de los imaginarios sociales actuales, tales como el individualismo y el materialismo, lo cual constituiría otro de los motivos por los cuales las conversiones religiosas comienzan a orientarse en México hacia este tipo de budismo.

Sin ir más lejos, la importancia que comienza a cobrar el budismo Soka en la región sudamericana, y en Argentina, en particular, puede observarse desde algunos datos estadísticos. Con la llegada de estas prácticas a la zona en la década de 1960, ya se habla de una tercera generación que comparte los principios fundamentales de esta filosofía de vida. Por debajo de Asia y Norteamérica, Sudamérica es el tercer continente con mayor cantidad de miembros afiliados a la Soka Gakkai, con aproximadamente 238.000 budistas ${ }^{4}$ (todos adscriptos en los últimos sesenta años). En cuanto al despliegue geográfico, en Argentina nos encontramos con una gran cantidad de centros Soka a lo

\footnotetext{
${ }^{4}$ A este número debe sumársele el insoslayable caudal de personas que, sin realizar el trámite burocrático para transformarse en miembro formal de la institución, acude a los encuentros, practica la filosofía de vida y comparte sus espacios para la realización de las actividades propuestas por el budismo Nichiren.
} 
largo y ancho del país: desde el Auditorio de la Paz en la Ciudad Autónoma de Buenos Aires, pasando por los centros de Castelar, Temperley y Mar del Plata- todos en la provincia de Buenos Aires- y también llegando a las provincias de Córdoba, Santa Fe, Neuquén y Tucumán.

Es en el marco de estas preocupaciones que se inserta nuestro interés por la dimensión del género: ¿Cómo se posiciona este budismo, centrado en el individuo y su capacidad de iluminación, frente a la diferencia y las desigualdades entre varones y mujeres? ¿Cuál es la materialización institucional de tal posicionamiento, en un marco discursivo que reafirma la dignidad humana? ¿A través de qué mecanismos refuerza los estereotipos que adopta? Retomamos aquí indagaciones previas que, si bien analizan otros budismos y desde una perspectiva que no atiende especialmente el rol institucional, nos han servido para identificar posibles claves analíticas.

Alice Collett (2014) propone dos niveles de análisis, el de la ética y el de la práctica, y sostiene que la filosofía budista -en sus múltiples ramas- no es necesariamente machista ni progresista en cuestiones de género, sino que los contextos específicos en los cuales se la practica son los que explican la discriminación de las mujeres. Su estrategia consiste en avanzar en dos operaciones complementarias, que son el análisis de los Sutras -en tanto portadores de la doctrina- y la historización -que permite corroborar el carácter situado de las formas de discriminación de género dentro de la práctica-. Además, llama la atención sobre una serie de riesgos y deficiencias que ha habido a la hora de describir el fenómeno (Collett, 2006), a saber: 1 - la tendencia a poner el lente sobre dos Sutras, habiendo al menos once que abordan el tópico; 2- el carácter insuficiente del análisis de las escrituras para saldar la discusión que es, al mismo tiempo, ética y práctica; 3- los riesgos que conlleva occidentalizar los análisis y, por lo tanto, descontextualizarlos. Liz Wilson, por su lado, propone estudiar la evolución del activismo en relación con el budismo, la evolución del feminismo en relación con el activismo budista y de mujeres en particular, y el debate académico en relación con el feminismo y las mujeres en el budismo (Wilson, 2012). Al evaluar estos tres planos conjuntamente, la autora logra empalmar una historización del campo disciplinar de estudios de género con el budismo, el activismo de mujeres budistas, y los movimientos sociales de género, produciendo una perspectiva más amplia.

La intersección entre género y budismo no es, pues, un tópico novel dentro de la literatura especializada. Incorporamos de nuestras predecesoras el llamado a no reducir la cantidad de dimensiones de análisis, al tiempo que reconocemos el carácter complejo del fenómeno: para dar cuenta de la relación entre varones y mujeres practicantes de budismo, el estudio de la filosofía no es suficiente. La propagación de las enseñanzas se da en un lugar y tiempo histórico específico, en una sociedad con posiciones tomadas o en movimiento sobre la cuestión de género, a través de una institución que reviste sus estereotipos y tiene mecanismos propios de (re)producir relaciones entre sus miembros. No contamos hoy, sin embargo, con investigaciones que aborden el fenómeno para el caso de este budismo en particular, en esta región del mundo, y desde una perspectiva 
institucional. Los trabajos sobre budismo Soka en América Latina no se han ocupado del género, ni tampoco han prestado especial atención a las cuestiones organizativas de la institución, situación que se hizo evidente en la investigación al intentar reponer organigramas, jerarquías y esquemas de distribución de tareas.

Nos interesa, como perspectiva analítica, la relación contingente entre desigualdad y diferencia conceptualizada por Rogers Brubaker (2015):

[las diferencias de raza, etnicidad, idioma, religión, género, sexualidad, ciudadanía] no están intrínsecamente ligadas a la desigualdad; diferente no necesariamente implica desigual. La relación entre diferencia y desigualdad es contingente, no necesaria; es empírica, no conceptual. $\mathrm{Y}$ el grado y la manera en que la desigualdad se estructura varía en el tiempo y según los contextos. (p. 11)

Las desigualdades entre varones y mujeres en la Soka-y cualquier otro espacio institucional- deben ser analizadas, entendemos, teniendo como punto de partida su carácter contingente y contexto-dependiente. Esta propuesta implica poner el foco sobre la construcción y articulación de las desigualdades, que es eminentemente empírica y se entrelaza con dimensiones simbólicas. Siguiendo la definición de género de Scott (1986) como construcción social de la diferencia sexual, nos preguntamos: ¿Qué desigualdadesentre varones y mujeres se producen enla SGIAR a partir de dicha diferencia sexual?? ¿Cómo son (re)producidas? ¿Existen paradojas? ¿Habilita la institución espacios que permitan establecer una igualdad entre los distintos actores sin que ello recaiga en una desdiferenciación?

Estas desigualdades se articulan en un enclave específico. En Argentina, a lo largo de las últimas décadas, han proliferadodemandas de movimientos feministas, de mujeres y de diversidad sexual. Si bien no es el objeto de este trabajo, es sabido que dichas demandas han suscitado una reacción neoconservadora.Esta última reviste un insoslayable componente religioso que interviene de lleno en el campo de la política democrática.Es que, en efecto, la comprensión de los posicionamientosde las instituciones secularesfrente al cambio social en materia de género y sexualidades no puede prescindir de la consideración de la dimensión sociopolítica(Vaggione y Machado, 2019).

Considerando todo lo anterior, el presente artículo describe y analiza el budismo Nichiren en Argentina motivado por preguntas referentes al género, lo cual nos llevó a producir una serie de datos institucionales/organizacionales que son los que aquí presentamos. Estos se desprenden de un proceso de investigación que se valió de múltiples técnicas y se desarrolló a lo largo de tres años (2018-2020), cuyas especificidades serán abordadas en el apartado metodológico que sigue a continuación.

En lo que respecta al despliegue del análisis, la sección estará dividida en cinco partes. Cada una de ellas dará cuenta de distintos rasgos institucionales que producen y reproducen estereotipos de género específicos, cristalizándose una diferencia jerarquizada entre varones y mujeres. Estas partes abordan: 1- la distribución de cargos 
de alta jerarquía en la institución a nivel nacional e internacional, en que se observa la preeminencia de los hombres; 2- los criterios de clasificación empleados para la confección de grupos -que son sexo y grupo etario/edad- y las actividades asignadas a cada uno de ellos, en donde pueden observarse los estereotipos dominantes y la menor autonomía de las mujeres (que pasan de jóvenes a adultas cuando forman familia y adoptan el rol de madre, mientras que los hombres mantienen mayor libertad para decidir el momento transicional); 3- el modo en el que el género se imprime en el espacio físico, al existir un centro cultural de la Soka dedicado especialmente a las mujeres, sobre el cual es posible indagar para describir los estereotipos predominantes; 4- el concepto de mujer representado en el diario de la organización, escrito principalmente por varones, que se construye como sinónimo funcional y simbólico de madre; 5- el estereotipo de hombre, rastreado tanto en el periódico institucional como en sub-grupos, asociado a la fuerza y figurado como columna vertebral de la organización.

\section{Enfoque y metodología}

Para analizar los modos en que opera la dimensión de género al interior del budismo Nichiren en Argentina empleamos la combinación de distintas técnicas cualitativas, las cuales enmarcamos dentro de un abordaje etnográfico. Entre estas técnicas se encuentran la observación participante, las entrevistas en profundidad, y el análisis hermenéutico documental; en su diversidad, todas ellas aplicadas para desplegar el fenómeno de interés, al servicio de producir datos y descripciones (Denzin y Lincoln, 2011). Dicha producción fue llevada adelante en el contexto de una investigación colaborativa, en el marco de un proyecto de investigación que enriqueció el producto final con múltiples y diversas miradas. En el equipo confluimos investigadores/as de distintas disciplinas, con diferentes trayectorias e intereses: cuatro varones y tres mujeres, de entre 25 y 51 años de edad, formados/as en sociología, antropología social, filosofía y ciencias de la educación. Dentro del proyecto marco, uno de los colectivos a analizar era el budista que, por la adscripción previa de dos integrantes del equipo, acabó por circunscribirse al budismo del Sutra del loto en el Área Metropolitana de Buenos Aires (nucleado en la SGIAR).

Comencemos por señalar que es un aspecto ventajoso de la etnografía el uso de diversas fuentes, dado que amplían los tópicos de análisis y maximiza el volumen de datos (Hammersley y Atkinson, 1994). Entendemos por etnografía un enfoque vinculado a ciertas técnicas, una preocupación por la reflexividad y la explicitación de esas dimensiones dentro de la escritura (Guber, 2011), que redunda en investigaciones de tipo inductivo-iterativo cuyas hipótesis iniciales suelen experimentar una metamorfosis al atravesar el trabajo de campo (O’Reilly, 2005). Sin ir más lejos, la pregunta que articula nuestra reflexión, referida a los modos en que opera la dimensión de género al interior del grupo social y la organización analizada, resultó justamente del trabajo de campo. Las jerarquías diferenciales entre varones y mujeres no estabanproblematizadas en la literatura sobre budismo y nuevas espiritualidades a escala argentina o latinoamericana, 
tampoco formaba parte de nuestros objetivos previos, sino que se fue consolidando como pregunta a medida y por causa de experiencias situadas en las interacciones de campo. En la misma dirección, las herramientas analíticas y estrategias de indagación fueron pensadas y activadas en el intercambio con nuestros/as interlocutores/as; en términos de Rosana Guber (2020), la teoría está vista desde el campo y es allí donde se suscita, se activa y se transforma.

A poco tiempo de comenzar la investigación, en 2018, la cuestión de género se consolidó como una fuente de preguntas: ¿Cómo se estructura el organigrama de la Soka? ¿Por qué los lugares de preeminencia y autoridad han sido ocupados siempre por varones? ¿Cuáles son las implicancias de que las tareas entre los/as practicantes se distribuyan según criterios de sexo y edad -varones jóvenes, señores, mujeres jóvenes, señoras-? ¿Qué tipos de actividades recaen sobre cada una de las cuatro categorías? ¿Se encuentran estas actividades ancladas en algún estereotipo sexo-genérico? ¿Por qué hay un Centro Soka de Mujeres ${ }^{5}$ y cuáles son sus características?

Estas preguntas y muchas otras, emergentes de la perplejidad al hacer investigación de campo, no estaban contempladas en la versión inicial del proyecto de investigación. En este sentido, resulta adecuada la caracterización de la etnografía sociológica propuesta por Esteban Benzecry, quien sostiene que la principal característica del enfoque consiste en acercarse al campo armados con un casco de teorías flexibles e intercambiables y, a partir del trabajo de campo (no antes), determinar qué preguntas podemos contestar con los datos que hemos producido (2017). En suma, la aproximación al budismo del Sutra del loto y los vínculos e involucramientos allí producidos alumbraron las inquietudes por los roles de varones y mujeres, tópico sobre el cual hay un vacío en la literatura y, paradójicamente, abundan los datos.

En orden de aplicación, las técnicas fueron la observación participante, la realización de entrevistas en profundidad, y el análisis documental. En lo que refiere a la observación participante, es oportuno señalar que se llevó adelante por varios/as integrantes del equipo de investigación, con distintos grados de implicación previa con el objeto de estudio, que iban desde el desconocimiento total de la práctica budista hasta la participación activa a nivel institucional. El grado de implicación y sus efectos sobre los datos producidos fueron supervisados con atención a partir de una puesta en común reflexiva y colectiva periódica, en un proceso continuo de objetivación participante (Bourdieu, 1978: 67-69). Concretamente, la observación participante consistió, para quienes no estaban previamente familiarizados con el budismo, en la incorporación a los hanes $^{6}$ y las actividades y eventos impulsados por la SGIAR: en su sede Central

\footnotetext{
5 Adyacente a la sede central de la institución, este centro fue inaugurado en 2016 para homenajear y respetar a las mujeres, "hacer la causa para cambiar el karma de la mujer en la sociedad" (Entrevistada 20) es decir, cambiar su papel sumiso y sufriente.

${ }^{6}$ El han es la unidad territorial mínima en la que se descomponen los rituales budistas. Estos constan de departamentos o casas de algunos practicantes que puedan albergar semanalmente a
} 
(conocida como kaikán ${ }^{7}$ ), se participó de diferentes actividades artísticas (coros, ensambles musicales y grupos de baile) en vista a un festival realizado en marzo del 2019, en el recinto de Tecnópolis, un espacio para la realización de eventos de dimensiones considerables ${ }^{8}$. En este último evento el etnógrafo se desempeñó en uno de los grupos expositores del festival, en carácter de participante pleno.

Tras 18 meses de trabajo de campo, y contando ya con la dimensión de género como eje de indagación, una integrante del equipo (que hasta ese momento había accedido al budismo exclusivamente a partir de notas de campo de otros miembros y bibliografía especializada), se acercó al Centro Soka de Mujeres por la Paz, lugar en el que hizo un recorrido acompañado de una nativa, a quién además realizó una entrevista informal -o no solicitada previamente- (Hammersley y Atkinson, 1994). Las observaciones y análisis que emergen de ese espacio físico y la forma en que los/as practicantes lo explican es tematizado en una sección del artículo.

Posteriormente se administraron entrevistas en profundidad a miembros $(n=25)$ de distintas edades, desde niños/as hasta adultos/as mayores; una fue administrada a un grupo familiar en su conjunto (pareja junto con hijos/as). Es menester señalar que el contacto con las/os entrevistadas/os fue posible gracias a la ayuda de dos informantes clave (Hammersley y Atkinson, 1994), cuya doble adscripción -al campo budista y al de las ciencias sociales- resultó decisiva a lo largo del proceso de investigación. Se guardaron en todos los casos los reparos éticos correspondientes, explicando previamente las características sustantivas e institucionales/administrativas del proyecto de investigación.

Las entrevistas estuvieron atravesadas por los avatares de la situación epidemiológica en el país: a partir de mayo de 2020 la implementación en Argentina del aislamiento social preventivo y obligatorio (ASPO) nos llevó a seguir adelante cambiando la modalidad presencial $(n=5)$ por la remota a través de redes sociales digitales $(n=20)$. Sin embargo, esto no afectó significativamente la dinámica o el tipo de datos producidos. En ambos casos el promedio de duración rondó los 40 minutos y fueron abordados los tópicos de interés. Se buscó comprender los modos en que los propios sujetos conciben la construcción social de la diferencia sexual (en otras palabras, el género) dentro del budismo, lo cual derivó en dos cauces: los/as entrevistados/as tendieron a diferenciar, por

todos los budistas que viven en un área de proximidad para poder así entonar conjuntamente el Sutra del Loto y desarrollar estudios y diálogos vinculados con la práctica.

7 También conocido como "Auditorio de la Paz", este centro cuenta con un gran auditorio con capacidad para centenares de personas. Si bien se utiliza para realizar las invocaciones del Sutra del Loto así como para profundizar el estudio de muchos principios budistas, también funciona como espacio en el que se preparan las diferentes partes de festivales, congresos y otros tipo de eventos.

${ }^{8}$ La Cumbre Soka de Jóvenes por la Paz se realizó el domingo 10 de marzo del 2019 en uno de los pabellones de Tecnópolis, en la provincia de Buenos Aires. Con frondosa concurrencia y el objetivo de difundir la filosofía budista desde el espacio juvenil de la institución, este evento se prolongó durante todo el día. 
un lado, la forma en que el género es (re)producido a nivel de la organización y, por otro, lo que el budismo como doctrina enseña de las relaciones entre seres humanos -varones y mujeres incluidos-. Si bien los materiales producidos en esta instancia fueron centrales, estos son tratados en profundidad en otro artículo (Sciurano, Alquezar y Melamud, 2022) consagrado a los modos de clasificar y co-hacer de los/as practicantes frente a lo que la Soka propone, mientras que aquí nos centramos específicamente en los elementos institucionales cristalizados.

Siguiendo a Malinowski (1986), partimos de distinguir entre lo que las personas dicen, lo que las personas hacen y lo que dicen acerca de lo que hacen, lo cual no necesariamente converge, pero está siempre en diálogo y puede resultar ilustrativo de las prácticas y valores predominantes en un grupo social. En tal sentido, el autor señala la necesidad de situar el análisis en varias escalas: la de las leyes y normas, la de las prácticas, y la de los discursos. En el esquema inicial, la observación participante apuntó a capturar las prácticas y contextos en los cuales se (re)producen o se subvierten los estereotipos de género; las entrevistas en profundidad, por otro lado, permitirían aprehender los discursos, esto es, los modos en que los/as propios practicantes dan cuenta del fenómeno. Queda pendiente, entonces, el análisis de las normas: ¿Cómo acceder a ellas?

Resultó sintomático, a nuestro criterio, la falta de investigaciones locales que se hayan centrado en el tema, sobre todo si consideramos la confluencia de abundantes indicios e interés social generalizado en torno a la problemática de género. La explicación que proponemos, desprendida del trabajo de campo, es la opacidad de la propia institución en lo que refiere a su lógica en general y, de forma concomitante, al lugar de la diferencia entre varones y mujeres dentro de la misma. Lidiar con esta dificultad no fue fácil en términos metodológicos. Hemos optado por tomarla como dato y aprovechar la variedad de fuentes disponibles, reconociendo paralelamente los huecos y los datos faltantes. Como se verá más adelante, las entrevistas a miembros fueron imprescindibles para prefigurar el funcionamiento institucional y nos permitieron identificar las normas.

A esta opacidad se suma una segunda dificultad, que es la multiplicidad de escalas entrelazadas e interdependientes que intervienen en y son constitutivas del fenómeno analizado. Nuevamente, esta vez siguiendo a Elizabeth Jelin, tomamos como fuente de datos la complejidad de los entrelazamientos, y colocamos el foco sobre ellos (Jelin, 2009). La SGIAR forma parte de la SGI, lo que nuestros/as interlocutores/s nos dicen sobre la práctica y su dimensión de género se encuentra atravesado tanto por las implicancias del quehacer a escala de interacciones microsociales, como por los textos de Ikeda que leen y discuten en los hanes, y los mensajes con frases y reflexiones que reciben periódicamente en sus teléfonos celulares. En suma, la institución y sus normas encarnan (de maneras diversas) en y son hechas por las personas de carne y hueso con las que hicimos trabajo de campo en el AMBA. 
Finalmente, utilizamos los Seikyos ${ }^{9}$, con el fin de rastrear los estereotipos de varones y mujeres. Estos documentos, en tanto textos escritos producidos por la organización que observamos, constituyen datos cualitativos y son pasibles de ser analizados del mismo modo que haríamos un análisis de entrevistas -a partir de una descripción, el análisis temático y la posterior interpretación- (Mejía Navarrete, 2011). Los diarios que nos ocupan circulan entre los/as miembros y contienen editoriales que han sido de gran utilidad, tanto por su contenido como por su presentación (tipografía, fotografías, etc.).

\section{La organización desde una perspectiva de género}

A la hora de abordar desde una perspectiva de género las maneras en las que el budismo Nichiren se despliega en Argentina, es preciso recordar algunos aspectos de la composición organizativa e institucional sobre la cual esta filosofía de vida se asienta. Fundada en noviembre de 1930 en Japón como SokaKyoiku Gakkai por TsunesaburoMakiguchi (quien fuera su primer presidente), la Soka Gakkai se estableció como la cabeza institucional que dirige y promueve la filosofía humanista del budismo Nichiren. Internacionalizada oficialmente para el año 1975, la entidad aquí presentada ya contaba con representación en Argentina desde 1963. Fue a partir de ese proceso que se terminó bautizando la Soka Gakkai Internacional Argentina (SGIAR). Con sede central en el barrio porteño de Villa Urquiza, en donde se encuentran el Auditorio de la Paz y más recientemente el Centro Soka de Mujeres por la Paz, el budismo del Sutra del Loto pasó a tener personería jurídica, así como también a intensificar progresivamente su presencia burocrática a lo largo y ancho de la geografía nacional.

Si bien el objetivo de este trabajo esel análisis institucional, es sabido que las instituciones no operan en un vacío. Por tal motivo resulta oportuno considerar el contexto sociocultural en relación con las cuestiones de género en Argentina a la hora de producir los datos aquí presentados. El trabajo de campo sobre laSGIAR sellevó adelante en un escenario de efervescencia de los movimientos de mujeres y de diversidad sexual; tal como señala Eleonor Faur, el género está en el aire (Faur, 2017), pudiendo identificarse como hitos la marcha del "Ni una Menos" en 2015, la lucha por la legalización de la interrupción voluntaria del embarazo visibilizada particularmente a partir del 2018, entre otros (Sciurano, Martínez Albanesi yNardacchione, 2021).

En lo que sigue a continuación desplegamos el análisis en cinco partes. La primera reseña sumariamente la participación de varones y mujeres en los cargos o puestos de alta jerarquía. La segunda describe los criterios de clasificación aplicados a la hora de construir grupos y asignar tareas. La tercera analiza la especificidad de los espacios físicos de la institución dedicados a homenajear a las mujeres. La cuarta repone,

\footnotetext{
${ }^{9}$ Estas publicaciones quincenales de la organización que combinan factura local con artículos que emanan del corazón mismo de la SGI tienen un rol central en la práctica, siendo el pilar esencial desde el cual se realizan estudios y diálogos en diversas instancias de socialización budista.
} 
a partir del análisis de los Seikyos, el estereotipo de mujer movilizado a nivel de la organización. La quinta y última parte analiza el estereotipo asociado a los varones, utilizando como insumo el análisis de los Seikyos, así como también las interacciones de un subgrupo de la Soka integrado exclusivamente por hombres.

\section{Distribución de varones y mujeres en altas jerarquías}

Partiendo del ámbito internacional, se puede realizar una afirmación que no da lugar a ambigüedades: los tres presidentes que tuvo la SGI a lo largo de su historia han sido hombres japoneses. Empezando por TsunaseburoMakiguchi (1871-1944), como fundador; continuando con Josei Toda (1900-1958), como el primer sucesor; y llegando a la actualidad con Daisaku Ikeda (1928), quien sigue ocupando tal cargo. Como se puede vislumbrar, ninguna mujer ha ocupado el cargo presidencial en la institución budista a nivel internacional. A su vez, varios practicantes nos fueron comentando a lo largo del trabajo de campo que la sucesión de lkeda no está prevista para ninguna persona en particular: ningún hombre o mujer ocupará el cargo de presidencia en el futuro cercano, sino que "la totalidad de la juventud heredará el compromiso de presidir a esta institución internacional" (Entrevistado 6).

Para entender cómo se distribuyen las responsabilidades y los cargos de la SGIAR, es menester considerar el criterio geográfico. Esto se debe a que las actividades budistas se desintegran en diferentes niveles de alcance territorial, comenzando por los hanes, pasando por zonas barriales o distritales (según sean en C.A.B.A o no), partidos, jurisdicciones, hasta llegar a las provincias y al nivel nacional. Las actividades ejecutivas y administrativas de los primeros niveles mencionados suelen estar distribuidas indistintamente entre varones y mujeres: ya sea como líder de han, responsable zonal o representante jurisdiccional, este nivel presenta una distribución equilibrada. Ahora bien, tal como comenta un informante clave, cuanto más se sube en la escala territorial, mayor es la probabilidad de encontrar hombres ocupando posiciones de toma de decisiones:

Cuando se pasa a una instancia mayor, superior, tienden a ser hombres, es decir: responsable de distrito, más que distrito, más arriba de jurisdicción y demás, partido, por ejemplo, ahí en general yo he visto siempre hombres... es como que el círculo va más arriba y en general se ven hombres, después a un nivel medio yo veo como una participación desde han hasta distrito... una cosa simétrica. (Entrevistado 23)

Resulta de especial interés este testimonio, proveniente de un practicante implicado en la organización, ya que ha resultado difícil (si no imposible) conseguir datos oficiales referentes a la ocupación de cargos institucionales. No hay documentos $u$ organigramas de acceso público que faciliten comprender los diferentes cargos que despliega la SGIAR a lo largo del país. En ninguna página de Internet, incluyendo la de la propia entidad argentina, nos encontramos con aquellos datos que pueden resultar de interés a la hora de dar cuenta del funcionamiento de los engranajes directivos. Este 
rasgo, sin embargo, no responde a una política global de la institución: en el caso de otros países (por ejemplo: Perú, República Dominicana, Estados Unidos o Venezuela) fue posible dar fácilmente con estos datos administrativos.

\section{Criterios de clasificación de practicantes y distribución de tareas}

Pasando ahora al análisis de los criterios que utiliza la institución para clasificar a los/as practicantes, en función de los que se distribuyen las tareas, encontramos dos ejes: el sexo y el pasaje a la adultez -ligado al estereotipo de género-. Más allá de las actividades conjuntas, el budismo Nichiren aboga por la constitución de núcleos identitarios en los que se pueden realizar actividades específicas entre miembros que tengan ciertas variables comunes. Por ello podemos hablar de categorías divisionales: señores o caballeros, señoras o damas, jóvenes varones, jóvenes mujeres y la división futuro (que se compone de niños/as generalmente menores a 15 años, independientemente de su sexo).

Si bien la institución organiza actividades de diversa índole guiada por la existencia de experiencias de vida plurales, cabe destacar un dato central: la manera en la cual un joven varón pasa a ser un señor no es la misma que la que una mujer tiene que desarrollar para realizar aquella transferencia de una categoría a otra. Aunque sea común que los varones cumplan cierta edad (aproximadamente entre los 35 y los 40 años) para dejar de ser considerados como jóvenes y pasar a ser señores, estos tienen una decisión totalmente autónoma para realizar tal pasaje; depende de sus intenciones y autopercepción como jóvenes o señores para abandonar una categoría y pasar a formar parte de otra. En el caso de las mujeres, si bien también existe la posibilidad de que realicen el traspaso a partir de la manera en la cual se consideren a ellas mismas, se debe resaltar un punto crucial: la maternidad implica, necesariamente, el pasaje de categoría. Esto trae consigo la reproducción de un trato diferencial entre la población masculina y femenina de la Soka. Mientras el hombre supedita su pasaje de una división a otra por un acto de volición, las mujeres tienen una condición que las limita en su elección, siendo que los mecanismos de "crecimiento" pasarán a estar subyugados a la maternidad (proceso que no necesariamente sea de interés o posibilidad para todas). Cabe destacar que para obtener tal información debimos recurrir a entrevistas realizadas a miembros, ya que ni en la página institucional ni en otros documentos oficiales pudimos saltearnos la opacidad que recubre estos datos.

Si nos detenemos en la distribución de actividades o roles que se ejecutan en el kaikán, estas diferencias entre sexos profundizan su construcción como categorías sociales estereotipadas de manera particular. Si bien en los hanes y otras actividades muchas veces los roles no están formalmente establecidos, y esto lleva a que mujeres y varones realicen actividades de las mismas características -como liderar el canto del 
mantra ${ }^{10}$, organizar las actividades, dar discursos de sus vidas personales ${ }^{11}$, entre otras-, es distinto lo que ocurre en la distribución de tareas a mayor escala.

Apreciamos en el trabajo de observación participante, en las entrevistas y en los documentos estudiados que las mujeres suelen ser asociadas con actividades de cocina, preparación de viandas y limpieza, mientras que los hombres se encargan de la seguridad, la logística y el transporte. Aunque no haya una justificación explícita para tal división de tareas, las practicantes implicadas en las actividades son quienes se encargan de la provisión de alimentos para los/as jóvenes que van a realizar sus propias actividades, mientras que las guardias y el transporte de personas y objetos siempre recaen sobre los hombres. Cabe aclarar, de todas formas, que estas tareas son de enrolamiento voluntario y no hay obligación de realizarlas a la hora de empezar la práctica.

Para finalizar, es preciso señalar que las actividades y estereotipos asociados a las categorías descritas hasta aquí no están libres de controversias, siendo que las/os miembros de la organización adoptan -en más de una oportunidad- el rol de emprendedores del cambio. He aquí tres ejemplos concretos manifestados en distintas entrevistas:

Como que no son roles estáticos de que el responsable es hombre y la que sigue es mujer sino que todo el tiempo se está reviendo la cuestión, y a mí me alentó12 muchísimo en uno de los últimos festivales el grupo de baile, por ejemplo, ponéle 2017, estaba diferenciado las chicas que bailaban en pollera y los chicos que tenían pantalón y remera. Y se planteó puertas para adentro del grupo: ¿por qué tenemos que vestirnos diferente?, y había varios integrantes de ese grupo que no se identificaban con el estereotipo de mujer con pollera, hombre con pantalón y eso al día de hoy es... se cambió y en los últimos festivales se los ve a todos vestidos iguales, por ejemplo, o eligiendo cada uno cómo se quiere vestir. (Entrevistada 18)

Sí, hay muchos miembros de la división de jóvenes de nuestra edad que sí son transgénero y eso se respeta al máximo. Si es una chica transgénero, es una chica transgénero. Se respeta y va para la división de chicas. En ese sentido es cómo la persona se identifica (...) Creo que en todos los ámbitos se está tratando todavía de descubrir también cómo podemos hacer para que esa persona se

\footnotetext{
${ }^{10} \mathrm{Si}$ bien el Sutra del Loto es cantado por todas las personas que acuden al han, siempre hay una persona que se sienta delante del resto, frente al Gohonzon (objeto de devoción principal del budismo, siendo un diploma en el que se encuentran las escrituras del buda original) y tiende a comandar los tiempos del canto.

11 Otro momento importante de las reuniones es el del relato de las experiencias personales. Esto se debe a que, a partir del ejemplo y la conmoción, las historias de superación logradas por la aplicación de principios básicos del budismo permiten demostrar la eficacia de estos recursos.

12 El aliento es el principal modo de comunicación entre los practicantes budistas: a partir de un efusivo apoyo y una permanente arenga a superar obstáculos y cumplir nuevos objetivos personales, el carácter conjunto y colectivo de las actividades budistas es manifestado.
} 
sienta parte (...) Creo que la organización está tratando de darle la vuelta... son casos muy puntuales, ¿viste? (...) creo que todavía estamos ahí, también, en el camino... así como toda la sociedad está en el camino de incluir a esas personas de una manera en que ellas se sientan realmente parte. (Entrevistada 20)

Y en realidad, si bien no hay un criterio estático tiene que ver con esto, con la postura y con el momento también de la fe en el que cada uno esté. Pero no es estático, para nada, sí se vino como aggiornando un montón porque antes era...cuando venía esta estructura rígida por ahí de Japón, que cuando te casabas al día siguiente ya eras de la división de damas más o menos, entonces pasó toda una generación de chicas de la Soka que no querían casarse para no pasar a la división de señoras. Eso es casi como un mito urbano, mito organizativo, porque hay todo un grupo de chicas que se casaron a los treinta y pico y decían que con tal de no sentirse señora, no se querían casar, pero quedó como un chiste, no es una realidad. Ahora nosotros por ejemplo nos casamos en el 2018, yo estuve casi dos años más en la división juvenil, porque yo soy como de esta nueva era donde el casamiento no te egresa de la división juvenil y bueno, siendo madre ahora sí, y estoy contenta. (Entrevistada 21)

\section{La inscripción del género en el espacio físico}

Merece mención la existencia del Centro Soka de Mujeres por la Paz, construcción ubicada dentro del mismo recinto en el que se encuentra el kaikán. Aunque no figure información de este espacio en ninguno de los segmentos de la página institucional -a pesar de la insistencia de varios/as practicantes, que entienden que este sí existe y brinda información relevante, y en numerosas ocasiones nos invitaron a buscar allí más información-, las experiencias de trabajo de campo nos permitieron realizar una reflexión a propósito de tal Centro.

Más allá del nombre y de algunas actividades unisex, este espacio se encuentra consagrado a reconocer la importancia de la lucha de las mujeres para difundir el budismo Nichiren y lograr el Kosen-Rufu ${ }^{13}$. Aunque en muchos casos sus espacios son de uso común, también se realizan actividades específicas que los/as miembros vinculan al sexo femenino. A lo largo del 2019, se desarrolló en este Centro una propuesta llamada "Protagonistas de una Cultura de la Paz", junto a actrices amateurs que encarnaron a mujeres destacadas de la historia cumpliendo un rol en pos de la paz (como Ana Frank, Victoria Ocampo y Florence Nightingale). A su vez, al lado de estas actividades que evidenciaban la centralidad de personalidades femeninas influyentes a lo largo de la historia de la humanidad, el Centro también proveyó durante el evento mencionado talleres de belleza y cocina, así como "alientos de amor compasivo"-temática tratada en

\footnotetext{
${ }^{13}$ Bajo la consigna de la persecución de "la paz mundial desde la felicidad individual", el budismo Nichiren tiene como meta su expansión mundial y difusión total en las distintas sociedades globales. De allí surge el concepto de Kosen-Rufu, que aparece como su misión central.
} 
los próximos párrafos-. Nuevamente, cobra fuerza la relación entre un sexo y algunas actividades simbólicamente concebidas como parte de ese mismo grupo. Esta caracterización se refleja en uno de los testimonios que recoge el primer Seikyo de julio de ese año: "Me inspiró muchísimo saber que cuando una mujer se pone de pie, enciende la luz de su hogar, llevando paz, amor compasivo y armonía a su entorno más cercano." (Seikyo 01/07/2019, p. 13).

Tampoco podemos dejar de mencionar la consideración privilegiada que se tuvo durante el evento con la figura de Rosa Parks. Lo particular del valor que se le daba, estaba íntimamente relacionado a su rol de "madre del movimiento por los derechos civiles", demostrando nuevamente el estrecho lazo que existe, en la práctica, entre la figura de mujer y la de madre.

Para concluir con la descripción de tal espacio, no deja de ser notable la presencia de flores que, si bien también decoran el kaikán, aquí tienen una presencia mucho mayor y son utilizadas como decoración por los distintos salones del edificio: grabadas en los techos, paredes, dibujadas en cuadros; flores reales y artificiales e incluso en fotografías tomadas por el propio Ikeda.

\section{Las mujeres en los Seikyos y en los eventos: ¿Quién y cómo las describe?}

Otra parada obligatoria para seguir estudiando los modos en que la institución budista argentina interpela la problemática de género es la de los Seikyos. Estos diarios narran la actualidad de la práctica budista en el país, presentan casos concretos de vidas influidas por la filosofía en cuestión, conmemoran fechas importantes para los/as miembros y difunden mensajes que profesan figuras importantes a nivel mundial ${ }^{14}$. El Seikyo es una herramienta institucional que promueve y socializa discursos, valores y atribuciones a la hora de comprender los diferentes vericuetos por los que el género es descrito, entendido y constituido por el budismo Nichiren.

Uno de los principales aspectos que vislumbramos tras el estudio del material periodístico, es que la figura de la mujer es continuamente recuperada en las notas que se escriben. Ya sea como editorial, cartas de lkeda o una reseña de cierta actividad que se haya realizado, en este espacio se le dedica una gran cantidad de páginas a la cuestión femenina. Pero, para describir con mayor precisión, los documentos suelen estar dirigidos hacia las madres. Las mujeres son abordadas, en primera instancia, bajo una perspectiva maternal. Esto último impide una clara diferenciación entre los términos de "mujer" y "madre", que llegan a constituir equivalentes simbólicos: “...donde hay una mujer decidida a seguir avanzando, se crea una nueva historia de esperanza y de alegría; esa

\footnotetext{
${ }^{14} \mathrm{Si}$ bien estos diarios se descontinuaron en 2019, sus lecturas siguen siendo habituales entre los practicantes teniendo en cuenta, sobre todas las cosas, que su suspensión se realizó hace poco tiempo. Hoy en día, su reemplazo es la revista "Humanismo Soka", con papel fotográfico y de ediciones mensuales, pero con un contenido muy similar a su antecesor.
} 
es la maravillosa misión de las mujeres, madres nutricias de la vida" (Seikyo 01/03/2016, p. 2).

Uno de los ejemplos más claros con respecto a esta utilización indistinta entre los términos mujery madre, lo encontramos en una nota publicada en la edición del 01/05/2018. Mientras la nota se titula "¡Que todos los días sean el día de las madres de la Soka!", el primer párrafo de la escritura (que es una carta enviada por Daisaku Ikeda), resalta la importancia de todas las mujeres Soka. Sin hacer disquisiciones, las palabras vuelven a enfocarse en el amor que una madre tiene por sus hijos como "foco irradiador de luz de felicidad" para volver a referirse a la totalidad de las mujeres que practican el budismo y que se encuentran organizadas en la División Femenina. Cerrando la carta vuelve a hacer referencia alternada, pero sin diferenciaciones entre las mujeres como integrantes aliadas en la Soka y las "laboriosas madres" que cumplen una misión fundamental en la sociedad mundial.

En cuanto a las principales características que se les atribuyen a las mujeres Soka -ya sea desde cartas escritas por Daisaku o Kaneko lkeda ${ }^{15}$, o reconstrucciones anónimas-, los términos más frecuentes son los siguientes ${ }^{16}$ : pureza, tolerancia, amor maternal y compasivo, calidez, vitalidad y paz. La idea de las mujeres como "personas que rechazan todo tipo de violencia", que buscan "nutrir y proteger la vida", y que "suavizan a la sociedad a partir de sus actos de bondad" también es reiterada. A esto se le debe añadir que suelen ser comparadas con distintos tipos de flores y, de hecho, las notas que están dedicadas a las mujeres suelen tener un diseño muy específico: fondos rosas con racimos y pétalos de decoración, acompañadas con ilustraciones de mujeres con bebés y niños a su alrededor.

Es interesante resaltar que la mayoría de las palabras orientadas a la especificación del rol, las características y las misiones de las mujeres se encuentran escritas por hombres 0 al menos basan las premisas en palabras de varones. Mayoritariamente, los alientos que buscan explicar a las mujeres su importancia dentro del budismo son propuestos por el presidente de la institución, Daisaku Ikeda. En otras ocasiones, son hombres especialistas o intelectuales los que establecen los principios básicos que toda mujer debe practicar para ser una verdadera budista. Uno de los casos más diáfanos, en este sentido, lo encontramos en la edición publicada el 01/07/2019. En una de las notas dedicadas a la conmemoración de la fundación del Departamento Femenino de la SGI, se recrea el diálogo entre dos hombres -Daisaku Ikeda y Adolfo Pérez Esquivel- para resaltar el rol crucial que la mujer tiene para el budismo Soka. En tal entrevista el presidente de la SGI afirma: "En el siglo XXI será necesario e, incluso,

\footnotetext{
15 Figura de referencia para las divisiones femeninas de la práctica budista a nivel mundial, la esposa de Daisaku Ikeda suele estar vinculada directamente con las actividades propias del ala femenina de la institución, interpelando continuamente a las mujeres que se comprometieron con la filosofía de vida en cuestión.

${ }^{16}$ Para conocer cuáles son los conceptos más utilizados en este material optamos por la utilización del atlas.ti, sistema informático de investigación cualitativa que permite el recuento de palabras sobre múltiples fuentes de modo simultáneo.
} 
imperioso contar con el poder espiritual de las mujeres; sobre todo, con su desinteresado afán de nutrir y proteger la vida, y su rotundo rechazo a todo tipo de violencia" (Seikyo 01/07/2019, p.12).

Este mecanismo es moneda corriente, mientras que son contadas las intervenciones que encontramos producidas por mujeres. Los diálogos entre lkeda y otros hombres que representan a la filosofía Soka, sean miembros o no, tienden a ser lo que más explican la importancia de las mujeres y de su misión particular.

En las pocas veces que hallamos a Kaneko Ikeda escribiendo, pudimos ver cómo privilegiaba lo que otros miembros hombres dictaminan acerca de la mujer, partiendo por su esposo hasta referirse a otras figuras masculinas de renombre. Parece más bien tener un rol de vocera de lkeda, sirviendo de mediadora entre las reflexiones de su cónyuge y las expectativas de las representantes de las divisiones femeninas (tanto señoras como jóvenes). De hecho, en los copetes de los artículos se presenta a Kaneko primero como esposa del presidente de la SGI, para luego aclarar su rol como titular honoraria de la División femenina.

Con el fin de clarificar lo que describimos anteriormente, podemos tomar algunos ejemplos a modo ilustrativo. En la edición del Seikyo del 15/02/2018, Kaneko comienza su carta haciendo referencia a su esposo: "Quisiera comenzar citando una estrofa de un poema que escribió mi esposo, 'A las grandiosas mujeres que viven con optimismo'." (Seikyo 15/02/2018, p.4).

A continuación, y sin adentrarse en sus pensamientos o palabras, se refiere nuevamente a su esposo cuando comenta el agradecimiento infinito que éste tiene frente a las divisiones femeninas del budismo Soka. Resulta muy difícil encontrar palabras que provengan de la propia intención de Kaneko, ya que sus discursos no tienen nada de específico: contrariamente, parafrasea y recrea continuamente a Daisaku lkeda, y en menor medida a los ex-presidentes -todos varones- de la institución. Las citas a continuación corresponden, en su totalidad, a una misma carta de Kaneko:

"Para mi esposo es una gran felicidad haber celebrado este hito junto a los miembros del mundo" (p.4); "Mi esposo siempre agradece expresamente el trabajo de todas ustedes en este sentido"; (p.4), "Muy feliz de verlas, mi esposo comentó asombrado que eran la muestra viviente de las palabras del buda Nichiren..." (p.5); "Mi esposo elogió su labor decididamente y, además de orar por la felicidad de su familia, le envió este mensaje personal". (p.5).

\section{Los hombres, pilar de la institución}

Son escasas las veces en las que aparecen artículos relacionados al sexo masculino. Sin embargo, cuando estos aparecen suelen hacer referencia al rol de "pilar" y "columna vertebral" que tienen los hombres dentro de la institución. A pesar de que no se establezcan tantos términos descriptivos sobre este grupo -al menos en comparación con 
el femenino- sí es reiterada la mención de la misión central delegada a los hombres dentro de la práctica. Impartiendo armonía entre las demás divisiones y protegiendo a la Soka y a sus miembros es como los señores budistas pueden asegurar el éxito humanista y mantener unida a la sociedad en su conjunto: "Por eso, desde la redacción del Argentina Seikyo, queremos agradecer a nuestros pilares dorados que, ante cada adversidad, se yerguen como firmes puntales que sostienen el desarrollo no solo de nuestra organización sino de toda la sociedad." (Seikyo 15/06/2018, p.2)

Resulta oportuno, en este punto, dedicar unas palabras al grupo Cascada. A partir del material proveniente de los Seikyos y de algunas entrevistas, nos encontramos con un sub-grupo de varones dentro de la organización, compuesto por hombres mayores (señores). Para empezar, este grupo se caracteriza por realizar una actividad que suele estar reservada al hombre dentro del budismo: "hacer guardias". En ese sentido, se encargan de que las actividades funcionen normalmente y sin alteraciones a partir de un compromiso voluntario con sus quehaceres.

Tanto en las notas publicadas en distintos Seikyos como en algunas de las entrevistas realizadas, pudimos apreciar la manera que tienen de autopercibirse los integrantes del grupo en cuestión, lo cual es de suma importancia en el proceso de construcción de arquetipos de género. Para empezar, estos hombres se conciben a sí mismos como "el motor de la actividad" o "la fuerza motriz de la práctica". Además, no deja de ser notorio que este subgrupo tiene una insignia propia que no comparte con ningún otro espacio, y hasta confecciona indumentaria con sus propios logos como refuerzo de su núcleo identitario.

Por otro lado, vislumbramos cómo circulan ilustraciones en las cuales se ven distintos hombres vistiendo camperas pertenecientes a este grupo, con su logotipo específico, "manteniendo el orden" y "cuidando de los demás". En estas imágenes se coloca al hombre-cascada en el centro de la escena, dentro de algún departamento en el que se realizan las reuniones, rodeado de niños, mujeres y ancianos. Es interesante, por lo tanto, comprender cómo esta facción interna de los señores budistas realza las características asignadas a los hombres de la organización, llevándolas un poco más lejos que el resto.

Volviendo a las cualidades que habitualmente les son asignadas a los hombres, ya en general, podemos resaltar que permanentemente se los considera como "reyes" o "campeones" de la vida. Sin mostrar temor a nada, los hombres aparecen como el sostén que guía y permite que las actividades de la práctica se desarrollen normalmente. Según algunas de las palabras escritas en estas notas, pareciera existir una patente capacidad de liderazgo entre los varones que componen la Soka, siendo que gracias a sus cualidades intrínsecas y su protagonismo continuo los demás grupos pueden proseguir con sus misiones y objetivos: “¡Hombres de bravura, triunfen en esta ruta por la dicha de los demás, librando la heroica gesta de los campeones genuinos!" (Seikyo 01/03/2016, p. 3).A partir del despliegue de dimensiones realizado hasta aquí, es posible apreciarlas contradicciones que anidanen el budismo Soka por causa de la prevalencia de 
estereotipos de género tradicionales. A propósito de las complejas relaciones entre movimientos feministas y de diversidad sexual, religión y política en la Argentina contemporánea, se ha señalado con justicia que las instituciones religiosas no adoptan un posicionamiento uniformemente conservadory/o reaccionario: las religiones pueden ser, paradójicamente, un factor favorable al cambio social y político por medio del pluralismo de puntos de vista al interior de sus instituciones y la posibilidad de proyectar un discurso bifronte, incluso cuando están en juego las formas tradicionales de regular la sexualidad y la familia (Jones y Vaggione, 2015).En este sentido, si bien en el caso estudiado las jerarquías institucionales, la distribución de tareas y espacios físicos, los criterios de pasaje entre categorías y los discursos reproducen desigualdades entre varones y mujeres, no es menos cierto que el reconocimiento de la mujer, en tanto categoría significativa, habilita una plataforma privilegiada para la puesta en cuestión de estos estereotipos(Sciurano, Alquezar y Melamud, 2022), fenómeno quese observa cuando se redefinen los criterios de pasaje, la vestimenta a utilizaro las tareas a realizar.

\section{Reflexiones finales}

Hemos analizado hasta aquí algunas de las formas en las cuales la construcción social de la diferencia sexual se manifiesta en la SGIAR, principal institución budista de la Argentina y presencia local de una organización con proyección internacional. La

observación de lugares, documentos e interacciones ha permitido identificar los estereotipos dominantes de la sociedad que allí se (re)producen y sus realizaciones en la práctica, que conviven en una organización cuyo horizonte explícito es el respeto por la dignidad humana y la capacidad de toda persona de alcanzar la iluminación. Paradójicamente, mujeres y varones se ven representados de modos diferentes, con asimetrías vinculadas a laprevalencia de estereotipos sociales tradicionales.

Si bien la institución la hacen las personasy sus interacciones, también son insoslayables aquellas reglas y estructuras más "sedimentadas" en la forma de discursos oficiales y divisiones organizativas.El trabajo aquí presentado busca describir esta última faceta. Dada la falta de investigaciones que se aboquen al análisis organizacional de la Soka, particularmente en su vinculación con la dimensión degénero, consideramos oportuno y necesario llevar adelante taldescripción.Aunque la SGIARhabilita instancias que ayudan a la visibilizacióny la diferenciación entre varones y mujeres, concediendo incluso espacios físicos que permiten amalgamar la diversidad de características exhibidas por las personas practicantes,la descripción realizada pone en relievevarios límites. Los mismos, articulados institucionalmente, reproducen desigualdades que se expresan con fuerza en la escala jerárquica de la organización y la distribución de cargos y tareas. Asimismo, y en rigor,nos encontramos frente a un proceso de reproducción de estereotipos ancladosenla diferencia sexual, lo cualdifícilmente redunde enuna corrección del vigente diferencial jerarquizado. 
Nos interesa, para concluir, hacer referencia al episodio de campo que cristaliza esta paradoja y pone en relieve la construcción del género dominante en la institución: durante el trabajo de observación participante realizado en la Cumbre de Jóvenes por la Paz en Tecnópolis, el etnógrafo presenció un discurso realizado por dos intelectuales hombres y una mujer, en el cual se insistía en la importancia del movimiento feminista dentro del espacio transformador de la juventud, haciendo hincapié en las luchas simbólicas que las mujeres llevaban adelante. Ahora bien, tal como explican las notas de campo escritas por el investigador: "Al finalizar, los presentadores dijeron que tenían el gusto de anunciar la creación de un nuevo galardón que la SGI Argentina entregaría. Lo llamativo fue que, tras el fuerte discurso de sesgo feminista, a los dos hombres se les dio el premio mientras que la mujer recibió ni más ni menos que un racimo de flores." (Nota de campo 10-03-19).

\section{Bibliografía}

Benzecry, C. (2017). "What did we say they've said? Four encounters between theory, method and the production of data". Ethnography, 18(1), 2434. https://doi.org/10.1177/1466138115592423

Bourdieu, P. (1978). "Sur l'objectivationparticipante. Reponses a quelquesobjections." Actes de la recherche en sciences sociales 20/21: 67-69.

Brubaker, B. (2015). Grounds for difference. Cambridge: Harvard UniversityPress.

Carozzi, M. J. (1999). "La autonomía como religión: la nueva era." Alteridades, Número $18,19-38$.

Collett, A. (2006). "Buddhism and Gender: Reframing and Refocusing the Debate". Journal of Feminist Studies in Religion, 22(2), 55-84. http://www.jstor.org/stable/20487864

Collett, A. (2014). Women in Early Indian Buddhism: Comparative Textual Studies. (South Asia Research). New York: Oxford University Press.

Daishonin, N.(2009). Los escritos de NichirenDaishonin.Madrid: Herder. 
Denzin, N. y Lincoln, Y. (2011). "Introducción General. La investigación cualitativa como disciplina y como práctica". En El campo de la investigación cualitativa, complicado por Denzin, Norman y Lincoln, Yvonna, 43-100. México: Gedisa.

Faur, E. (2017).Mujeres y varones en la Argentina de hoy: Géneros en movimiento. Siglo Veintiuno Editores.

Gancedo, M. (2015). "Rostros de una diáspora. Comunidad japonesa y religiosidad en la Soka Gakkai Internacional (Argentina)". Horizontes antropológicos [online], vol.21, n.43, pp.183-210.

Guber, R. (2011).La etnografía. Método, campo y reflexividad. Buenos Aires: Siglo XXI.

Guber, R. (2020). "Pensar la investigación de campo desde Ibero-América: 4 líneas y 4 paradojas". AIBR, Revista de Antropología Iberoamericana, 15 (03): 439-462. DOI:10.11156/aibr.150302

Hammersley, M. y Atkinson, P. (1994).Etnografía. Métodos de investigación. Barcelona: Paidós.

Ikeda, D. (1982).El buda viviente. Buenos Aires: Emecé.

Ikeda, D. (1984). La vida, un enigma. Buenos Aires: Emecé.

Jelin, E. (2009). "Rosas transplantadas y el mito de Eldorado. Travesías en el tiempo, en el espacio, en la imagen y en el silencio." Revista Del Museo De Antropología, 2(1), 75-86. https://doi.org/10.31048/1852.4826.v2.n1.5408

Jones, D. y Vaggione, J. (2015). "Pluralismo religioso y políticas sexuales en Argentina". Religión e Incidencia Pública. Buenos Aires: Grupo de Estudios Multidisciplinarios en Religión. 
Lehmann, D. y Montalvo, M. G. (2019 [2004]). "La religión en las ciencias sociales contemporáneas en América Latina." Revista Mexicana de Sociología, Vol. 66, Número especial (Oct., 2004), 215-237.

Makiguchi, T. (2012 [1999]).Educación para una vida creativa. Buenos Aires: Editorial de la Universidad de Flores.

Malinowski, B. (1986 [1972]).Los argonautas del Pacífico Occidental. Barcelona: PlanetaDe Agostini.

May May, E. (2016). “¿Por qué me convertí? ¿Por qué se convirtieron? Los budistas Soka Gakkai en Mérida, Yucatán." Relaciones Estudios de Historia y Sociedad, 148 bis, pp. 205-241.

Mejía Navarrete, J. (2011). "Problemas centrales del análisis de datos cualitativos". Revista Latinoamericana de Metodología de la Investigación Social, 1, pp. 4760.

O'Reilly, K.(2005). Ethnographicmethods.Londres: Routledge.

Puig Zhamaganov, B. E. (2011).“BudismoNichiren: Origen, esencia, práctica y su presencia en Sudamérica." Ponencia presentada en XII Congreso Internacional de ALADAA, Asociación Latinoamericana de Estudios de Asia y África. Puebla, México, 13 al 15 de junio.

Semán, P. y Viotti, N. (2015). "El paraíso está dentro de nosotros. La espiritualidad de la Nueva Era, ayer y hoy." Nueva Sociedad, №260, 81-94.

Sciurano, G. A., Alquezar, M. \&Melamud, M. (2022). "Voces de la igualdad y la diferencia en el budismo Soka: El género como paradoja" [Manuscrito no publicado].Debate Feminista, 64(64).

Sciurano, G. A., Martínez Albanesi, L., \& Nardacchione, G. (2021). "Género, mujeres y contact improvisación: sobre el problema del límite y el rol distensor del discurso dentro del espacio pedagógico". Artseduca, 29(29), 157-169. 
Revista Punto Género N. 16 Diciembre de 2021

ISSN 2735-7473 / 145- 168

Scott, J. (1986). Gender: A Useful Category of Historical Analysis. The American Historical Review, 91(5), 1053-1075. doi:10.2307/1864376

Vaggione, J. y Machado, M. (2019).“Religious Patterns of Neoconservatism in Latin America”. Politics and Gender. Cambridge: Cambridge University Press.

Welsch, D. (2018). "Passing on the law. The Growth of Soka Gakkai in Argentina." International Journal of Latinamerican Religions, 2 (1), 22-40.

Wilson, L. (2012). "Buddhism and Gender," in Buddhism in the Modern World, editado por McMahon, David. Londres: Routledge. 